\title{
CEP-stable, few-cycle, kHz OPCPAs for attosecond science: Energy scaling and coherent sub-cycle pulse synthesis
}

\author{
K.-H. Hong, ${ }^{1}$ S.-W. Huang, ${ }^{1}$ J. Moses, ${ }^{1}$ X. Fu,${ }^{1}$ G. Cirmi,${ }^{1}$ C.-J. Lai, ${ }^{1}$ S. Bhardwaj,${ }^{1}$ and F. X. Kärtner ${ }^{1,2}$ \\ ${ }^{1}$ Department of Electrical Engineering and Computer Science and Research Laboratory of Electronics, \\ Massachusetts Institute of Technology (MIT), Cambridge, MA 02139, USA \\ ${ }^{2}$ DESY-Center for Free-Electron Laser Science and Hamburg University, Hamburg, Germany \\ *Email: kyunghan@mit.edu
}

High-energy, carrier-envelope phase (CEP)-stable, few-cycle optical pulses have been a major driving source for producing isolated attosecond pulses via high-order harmonic generation (HHG). Besides the Ti:sapphire chirped-pulse amplification (CPA) technology with external pulse compression technique, recently, ultrabroadband optical parametric chirped-pulse amplification (OPCPA) has been regarded as a very promising technology for next-generation attosecond science because of several unique features, such as wavelength coverage from the visible to the mid-infrared, energy scalability to well above the $\mathrm{mJ}$ level while maintaining few-cycle pulse durations, and low CEP drift during the amplification. Especially, mid-IR few-cycle OPCPA [1] can greatly extend the cut-off energy of HHG, enabling attosecond science at high photon energy approaching keV [2].

In this paper, we present 1) the energy scaling of a CEP-stable, few-cycle, $\mathrm{kHz}$ OPCPA at $2.1 \mu \mathrm{m}$ using a high-energy, ps, cryogenic Yb:YAG CPA laser [3] and 2) the coherent synthesis of its pulse with a CEP-stable, few-cycle, 800-nm pulse for sub-cycle pulse generation [4]. Fig. 1 shows the schematic of a 2.1- $\mu \mathrm{m}$ OPCPA system with two optically synchronized pump lasers, Nd:YLF $\left(1^{\text {st }}\right.$ and $2^{\text {nd }}$ stages $)$ and $\mathrm{Yb}: \mathrm{YAG}\left(3^{\text {rd }}\right.$ stage $)$ lasers. The seed is generated by intra-pulse difference-frequency generation from an octave-spanning Ti:sapphire oscillator and is thus passively CEP stabilized. The CEP-stable, 2.1- $\mu \mathrm{m}$ pulse was amplified to $0.8 \mathrm{~mJ}$ with a bandwidth of $>470 \mathrm{~nm}$, supporting 3.5-cycle duration. Compression was achieved using Brewster-cut Suprasil300 glass. Further OPCPA optimization and HHG experiments will be discussed. Secondly, to push the pulse duration down to the single-cycle limit by coherent pulse synthesis, we developed a CEP-stable, 3-cycle OPCPA at $800 \mathrm{~nm}$ pumped by a

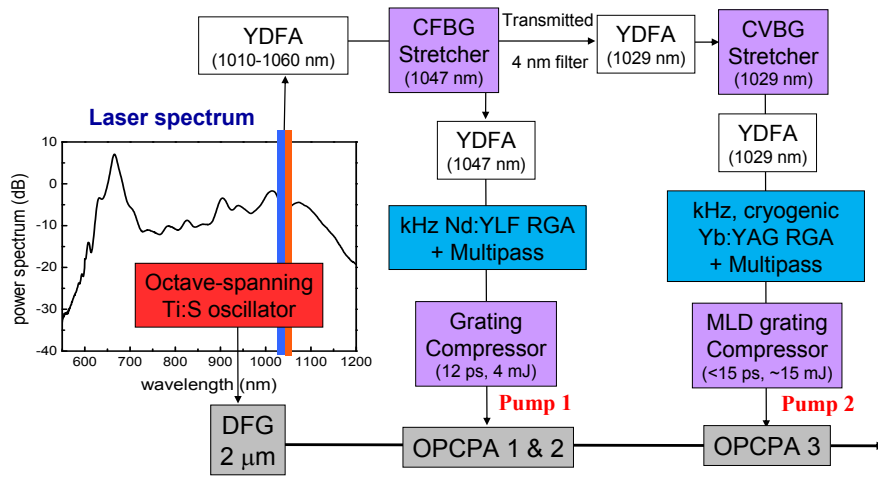

Fig. 1 Few-cycle, kHz, 2.1- $\mu \mathrm{m}$ OPCPA pumped by optically synchronized Nd:YLF and cryogenic Yb:YAG CPA lasers (YDFA, Yb-doped fiber amplifier; CFBG/CVBG, chirped fiber/volume Bragg grating; MLD, multi-layer dielectric; RGA, regenerative amplifier). frequency-doubled Nd:YLF laser. The compressed output from the second stage of the 2.1- $\mu \mathrm{m}$ OPCPA was combined with the $800-\mathrm{nm}$ pulse. A balanced crosscorrelator with a feedback loop locked the timing between two pulses with a jitter of $\sim 250$ as. The synthesized pulse duration of $\sim 0.6$ cycle was measured using two-dimensional spectral shearing inteferometry (2DSI) and the pulse energy was $15 \mu \mathrm{J}$, scalable to $\sim \mathrm{mJ}$ level using the cryogenic Yb:YAG pump laser. Simulations confirm the feasibility of direct isolated attosecond pulse generation using this waveform.

[1] T. Fuji et al., Opt. Lett. 31, 1103 (2006); X. Gu et al., Opt. Express 17, 62 (2009); J. Moses et al., Opt. Lett. 34, 1639 (2009).

[2] M. C. Chen et al., Phys. Rev. Lett. 105, 173901 (2010).

[3] K.-H. Hong et al., Opt. Lett. 35, 1752 (2010).

[4] S.-W. Huang et al., "Scalable High-Energy Sub-Cycle Waveform Synthesis for High-Field Physics," High Intensity Lasers and High Field Phenomena 2011 (Feb. 16-18, 2011, Istanbul, Turkey), JWC4. 